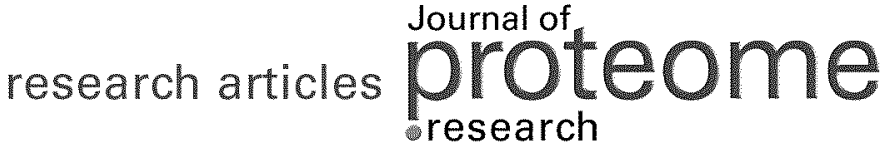

\section{Essential Core of Protein-Protein Interaction Network in Escherichia coli}

\author{
Chen-Ching Lin, ${ }^{+, \neq}$Hsueh-Fen Juan, ${ }^{\ddagger}$ Jen-Tsung Hsiang, ${ }^{\$}$ Yih-Chii Hwang, ${ }^{\dagger}$ Hirotada Mori, ${ }^{*, l l, \perp}$ \\ and Hsuan-Cheng Huang, ${ }^{*}$
}

\begin{abstract}
Institute of Biomedical Informatics, Center for Systems and Synthetic Biology, National Yang-Ming University, Taipei 112, Taiwan, Department of Life Science, Institute of Molecular and Cellular Biology, Graduate Institute of Biomedical Electronics and Bioinformatics, National Taiwan University, Taipei 106, Taiwan, Department of Physics, National Dong Hwa University, Hualien 974, Taiwan, Graduate School of Biological Sciences, Nara Institute of Science and Technology, Ikoma, Nara 630-0101, Japan, and Institute for Advanced Biosciences, Keio University, Tsuruoka, Yamagata 997-0017, Japan
\end{abstract}

Received October 19, 2008

\begin{abstract}
Essential genes are responsible for the viability of an organism. Global protein interaction network analysis provides an effective way to understand the relationships between protein products of genes. By means of large-scale identification of essential genes and protein-protein interactions, we investigated the substructure of the protein interaction network in Escherichia coli and identified all the cliques in the network. Our analysis showed that larger cliques tend to have larger fractions of proteins encoded by essential genes. By merging the maximum clique with overlapping neighboring cliques, we observed a dense core of the protein interaction network in Escherichia coli with significantly higher ratio of essential genes. The protein network of Saccharomyces cerevisiae also shows strong correlation between clique and essentiality, and there exist similar dense clusters with high essentiality. Our results indicated that the observed structure of essential cores might exist in higher organisms and play important roles in their respective protein networks.
\end{abstract}

Keywords: essential gene $\bullet$ protein-protein interaction network $\bullet$ clique $\bullet$ Escherichia coli

\section{Introduction}

In recent years, technological advances make possible the systematic mapping of protein-protein interaction, or interactome mapping. Experimental procedures such as yeast-two hybrid $^{1}$ and protein co-immunoprecipitation ${ }^{2}$ have been applied to generate large-scale protein interaction networks for most of the model organisms, including Saccharomyces cerevisiae $^{3,4}$ and Escherichia coli. ${ }^{5,6}$ Although constructing and understanding these networks are far from being completed, they have revealed global topological characteristics ${ }^{7}$ and dynamic features ${ }^{8}$ of protein interaction networks related to known biological properties. This accumulating information derived from large-scale protein interaction networks opens a window to understand biological organizations at the system level, but the coming challenges are to organize this information into models of cell machinery and to present interactome information with higher confidence. Understanding the bio-

* To whom correspondence should be addressed. Hirotada Mori: tel, +81743-72-5660; fax, +81-743-72-5669; e-mail, hmori@gtc.naist.jp. Hsuan-Cheng Huang: tel, +886-2-28267357; fax, +886-2-28202508; e-mail, hsuancheng@ ym.edu.tw.

${ }^{\dagger}$ National Yang-Ming University.

‡ National Taiwan University.

${ }^{\S}$ National Dong Hwa University.

" Nara Institute of Science and Technology.

${ }^{\perp}$ Keio University. logical organization at a system level is among the key objectives in the postgenomic era.

Gene deletion is usually applied to identify essential genes. ${ }^{9}$ Since not all functions of the deleted genes can be compensated by their existing counterparts, gene deletion may result in the demise of the organism. Hence those genes directly involved in organism lethality are called essential genes and the proteins encoded by them are called essential proteins. Several earlier single-gene-deletion experiments had identified certain essential genes in E. coli, ${ }^{10}$ Bacillus subtilis ${ }^{11}$ and S. cerevisiae, ${ }^{9,12}$ and the analysis of protein essentiality in a protein interaction network was made possible.

A protein interaction network can be abstracted as a mathematical graph, permitting analysis with graph theoretical algorithms. Proteins are represented as nodes in the graph and interactions are edges connecting the nodes. Most of the observed protein interaction networks are found to be scalefree, with a power-law distribution of the node connectivity. ${ }^{13,14}$ Such networks contain a small number of highly connected nodes, called hubs. Several authors have proposed that the topological properties of the protein interaction network could be strongly related to protein essentiality. Hubs are usually more essential than nonhubs. ${ }^{13,15}$ Proteins with high betweenness tend to be essential. ${ }^{16,17}$ However, other authors have taken an opposite stance, arguing that protein essentiality is poorly related to the topological properties of protein interac- 
tion networks. ${ }^{18-20}$ New large-scale measurements of essential genes $^{10}$ and protein-protein interactions ${ }^{5}$ in E. coli provide additional information to clarify this controversy.

On the other hand, analysis of subgraph structures in protein interaction networks can also give another perspective of the topological roles of essential proteins in the network. Previous studies suggested that biological systems are composed of interacting modules with different functions. ${ }^{21-23}$ Identifying those functional modules in a protein interaction network is pivotal to the understanding of the organization and interaction of cellular processes in both eukaryotes and prokaryotes. ${ }^{17,24}$ It has also been pointed out that protein complexes are generally associated with dense regions. ${ }^{25}$ Wuchty and Almaas ${ }^{26}$ showed a form of essential core, where nodes more centrally located in a core are more likely to be essential. Cliques, or complete subgraphs, are the densest regions in a network. However, the biological roles of cliques remain unclear. We proposed that, since highly connected nodes tend to be essential and essential genes or proteins are found to be enriched for interacting with other essential genes or proteins, ${ }^{15}$ cliques may also be associated with essentiality.

In this study, we investigated the subnetwork structure of the protein interaction network and developed an algorithm to identify all the cliques in the network as well as to merge overlapping cliques. Our results show a strong correlation between the cliques and the essential proteins, and we suggest that an essential core, a new topological characteristic, exists in the protein interaction network of E. coli and other organisms.

\section{Materials and Methods}

Experimental Data of Protein-Protein Interaction and Essential Gene Identification. Experimental measurements of the protein-protein interactions of $E$. coli K-12, acquired by His-tag pull-down assay, ${ }^{5}$ were used to construct the protein interaction network for analysis in this study. The protein interaction data of $S$. cerevisiae were downloaded from the DIP database. ${ }^{27}$ In a recent study, we identified 303 essential gene candidates in E. coli K-12 as genes unable to be deleted from the chromosome. ${ }^{10}$ These 303 genes were used as the list of essential genes for analysis in this study. The list of essential genes for yeast was downloaded from the Saccharomyces Genome Deletion Project (http://www-sequence.stanford.edu/ group/yeast_deletion_project/).

Network Analysis. A protein interaction network may be represented as an undirected graph $G(V, E)$ that consists of a set of nodes $V$ and a set of edges $E$. Each node $u \in V$ represents a unique protein, while each edge $(u, v) \in E$ represents an observed interaction between two proteins $u$ and $v{ }^{28}$ The size of a graph is given by the number of its nodes. The degree of a node indicates the number of observed interacting partners of a protein. A clique of size $n$ is denoted by $K_{n}$, and is a complete $n$-node subgraph, by which we mean that within a subgraph, each pair of nodes is connected by an edge. The number of the undirected edges in a complete graph of $n$ nodes is given by $(n, 2)=n(n-1) / 2$, where $(n, k)$ is the binomial coefficient. If a clique is not a subgraph of any other clique, then this clique is called a maximal clique. Among all the maximal cliques, the largest one is the maximum clique.

The path length between two nodes in a graph is the number of edges in a shortest path connecting them, which is also known as the geodesic distance. The eccentricity of a node in a connected graph is the maximum path length between $v$ and any other node in the graph. The maximum eccentricity can be thought of and is defined as the diameter of the graph. Similarly, the minimum eccentricity is the radius of the graph. If the eccentricity of a node is equal to the radius, this node is a central node of $G$.

The centrality of a node $v$ determines the relative importance or closeness of a node within the graph. For a graph with $n$ nodes, the betweenness $C_{B}(v)$ of a given node $v$ is defined as $C_{B}(\nu)=\sum_{s<t}\left[\sigma_{s t}(\nu) / \sigma_{s t}\right]$, with $s \neq v \neq t \in V$. Here, $\sigma_{s t}$ is the number of shortest paths from $s$ to $t$, and $\sigma_{s t}(\nu)$ is the number of shortest paths from $s$ to $t$ that also pass through the node $v$. If more shortest paths cross a given node, this node tends to have a higher score of betweenness. Closeness centrality describes how close a node within a graph is to the other nodes. A node that has higher closeness centrality tends to have shorter geodesic distances to other nodes within the graph such that it is closer to the center of the graph. Thus, closeness measures how easily other nodes can be reached from it, or how easily it can be reached from the other nodes, and is usually positively correlated with other measures, such as degree. For a graph with $n$ nodes, the closeness $C_{C}(v)$ for a node $v$ is defined as $C_{C}(v)=$ $\left[1 / \sum_{t} d(v, t)\right]$, where $t \neq v \in V$ and $d(v, t)$ is the geodesic distance between nodes $v$ and $t$, that is, the closeness centrality $C_{C}(v)$ for a node $v$ is the reciprocal of the sum of geodesic distances to all other nodes in the graph.

Clique Finding and Clique Merging. The problem of finding the maximum clique for a given graph is an NP-complete problem. ${ }^{29}$ Here, we proposed a novel and efficient algorithm to find the maximum clique. The algorithm first identifies all the smallest cliques, $K_{3}$, by examining whether there exists an edge between each pair of neighboring nodes for any given node, and recursively finds other larger cliques. Detailed information about our algorithm is given in Supporting Information S2. This algorithm is suitable for protein interaction networks or other scale-free networks, since the complexity of this subroutine is decided by the average degree of a network. As there are only a small number of high-degree nodes in a scale-free network, its average degree is commonly low.

We merged the maximum clique with overlapping neighboring cliques to form a larger dense cluster. Cliques that share more than half of their nodes with the maximum clique were defined as overlapping neighboring cliques. More details about the clique-merging algorithm and the determination of proper threshold for shared nodes are described in Supporting Information S2.

\section{Results and Discussion}

We constructed the protein interaction network of $E$. coli based on our previous large-scale measurements of proteinprotein interactions of $E$. coli $\mathrm{K}-12 .^{5}$ There are 3039 nodes (proteins) and 11477 edges (interactions) in the protein interaction network of $E$. coli. The diameter of this network is 9 and the radius is 5 . Among the 3039 proteins, 260 proteins are products of essential genes, and denoted as essential proteins.

Topological Features of Essential Proteins in the Protein Interaction Network of $\boldsymbol{E}$. coli. We compared the topological properties of essential and nonessential proteins, and summarized the results in Table 1 . We found that the degree of the essential proteins was significantly higher (17.0 in average) than the nonessential proteins (6.7 in average); the betweenness centrality was also significantly higher for essential proteins. These results are consistent with the observation in $S$. cerevisiae. ${ }^{16,30}$ However, unlike yeast, no difference in the clustering coefficients was observed between essential and 
Table 1. Comparison of the Topological Properties between Essential and Nonessential Proteins of E. coli; between the Maximum Clique $\left(K_{7}\right)$ and Randomly Selected 7-Node Subnetworks

\begin{tabular}{|c|c|c|c|c|c|c|c|}
\hline & ess. & noness. & $P$-value & $K_{7}$ & random & $Z$-score & $P$-value \\
\hline Degree & 17.0 & 6.7 & $8.2 \times 10^{-10}$ & 125.4 & 7.6 & 14.1 & $2 \times 10^{-4}$ \\
\hline Betweenness & 0.00240 & 0.00063 & $1.8 \times 10^{-4}$ & 0.02227 & 0.00079 & 7.1 & $4.6 \times 10^{-3}$ \\
\hline Closeness & 0.31 & 0.30 & 0.127 & 0.37 & 0.30 & 4.2 & 0.001 \\
\hline
\end{tabular}

Table 2. The Numbers of Found Maximal Cliques and the Corresponding Numbers of Nodes for Various Sizes of Cliques in the Protein Interaction Network of E. coli and S. cerevisiae

\begin{tabular}{clllllll}
\hline & \multicolumn{4}{c}{ E. coli } & & \multicolumn{3}{c}{ S. cerevisiae } \\
\cline { 2 - 4 } maximal $K_{n}$ & nodes & cliques & essentiality & & nodes & cliques & essentiality \\
\hline$K_{0}$ & 1712 & - & $7.1 \%$ & & 2867 & - & $12 \%$ \\
$K_{3}$ & 1062 & 2110 & $8.3 \%$ & & 1038 & 2248 & $21 \%$ \\
$K_{4}$ & 172 & 364 & $14 \%$ & & 398 & 933 & $35 \%$ \\
$K_{5}$ & 65 & 134 & $23 \%$ & & 246 & 712 & $44 \%$ \\
$K_{6}$ & 17 & 16 & $29 \%$ & & 148 & 341 & $44 \%$ \\
$K_{7}$ & 7 & 1 & $86 \%$ & & 104 & 75 & $59 \%$ \\
$K_{8}$ & - & - & - & & 22 & 22 & $73 \%$ \\
$K_{9}$ & - & - & - & & 40 & 19 & $70 \%$ \\
$K_{10}$ & - & - & - & & 10 & 1 & $90 \%$
\end{tabular}

Table 3. List of Interacting Partners of $\mathrm{YdiE}^{a}$

\begin{tabular}{lrrl}
\hline protein & \multicolumn{1}{c}{$\mathrm{p} I$} & essential & \multicolumn{1}{c}{ functional annotation } \\
\hline RpsI & 10.94 & Yes & 30S ribosomal subunit protein S9 \\
RplQ & 11.05 & Yes & 50S ribosomal subunit protein L17 \\
RpsD & 10.05 & Yes & 30S ribosomal subunit protein S4 \\
RpsK & 11.33 & Yes & 2 30S ribosomal subunit protein S11 \\
RpsM & 10.78 & Yes & 30S ribosomal subunit protein S13 \\
RpsC & 10.27 & Yes & 30S ribosomal subunit protein S3 \\
RpsB & 10.93 & Yes & 50S ribosomal subunit protein L2 \\
RplW & 9.94 & Yes & 50S ribosomal subunit protein L23 \\
RpsG & 10.37 & Yes & 30S ribosomal subunit protein S7 \\
RplA & 9.64 & No & 50S ribosomal subunit protein L1 \\
NadE & 5.41 & Yes & NAD synthetase, NH3/glutamine-dependent \\
YoeA & 5.42 & No & CP4-44 prophage predicted disrupted hemin \\
& & & or colicin receptor (pseudogene) \\
HtrG & 9.1 & No & predicted signal transduction protein \\
& & & (SH3 domain)
\end{tabular}

${ }^{a}$ There are 13 interacting partner proteins of the only nonessential protein, YdiE, in the maximum clique $K_{7}$ of E. coli. Most of them are essential ribosomal proteins.

nonessential proteins in E. coli. This seems to contradict our expectation that a node which is densely clustered around other nodes might play a more important role in the network and hence higher biological significance. However, this might be attributed to the simpler subcellular organization of prokaryotes or the noisy systematic measurements of protein-protein interactions (Supporting Information S1). To further explore the role of a protein in a denser region of the interaction network, we investigated other related topological features of essential and nonessential proteins and found that the subgraph feature of cliques was closely related to essentiality.

Identifying Cliques in the Protein Interaction Network. In graph theory, a clique is defined as a subnetwork whose nodes are connected with each other. Cliques are the densest subnetworks, so proteins in a clique interact tightly with one another. To identify all the possible cliques in the protein interaction network, we developed a novel and fast algorithm as described later (in Materials and Methods).

Efficient maximum-clique finding continues to be a problem in graph theory, even though many algorithms are readily available. Here, we proposed an algorithm that is particularly suitable for scale-free networks. This type of networks has a particular feature; that is, only a small number of nodes have very high degrees of connectivity. Several previous studies have shown that protein interaction networks are scale-free. ${ }^{13,14}$ This is reasonable because it is highly improbable for a protein to simultaneously interact with multiple partners in protein interaction networks, as a protein does not have multiple binding sites to interact with many partners at the same time. Instead, it is more often that only a few proteins share the same small number of binding sites of a given protein. ${ }^{31}$ Since protein interaction networks do not have a large number of high-degree nodes, this algorithm works exceptionally well.

Correlation between Cliques and Protein Essentiality. We counted the number of cliques of all sizes and calculated the essentiality of every kind of cliques, as listed in Table 2. Only the numbers of the maximal cliques (defined in Materials and Methods) are shown. The essentiality of a subnetwork is defined as the ratio of the essential proteins to all proteins in a subnetwork. We observed a strong correlation between clique size and essentiality. As shown in Figure 1A, the essentiality of
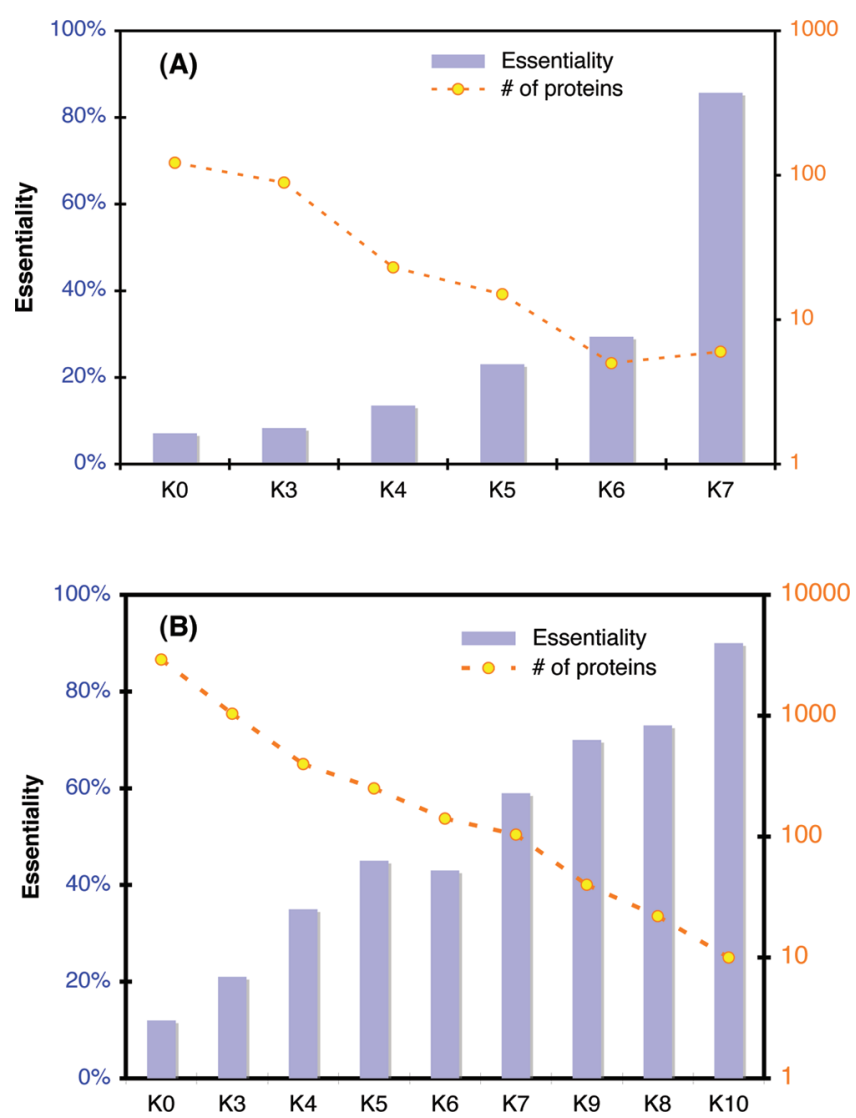

Figure 1. Distributions of the average proportion of essential proteins to the total number of proteins in different sizes of cliques within the protein interaction network of (A) E. coli and (B) S. cerevisiae. Larger cliques show higher essentiality than lower ones in both $E$. coli and $S$. cerevisiae. $K_{0}$ denotes the kind of proteins not forming any cliques. 
(A)

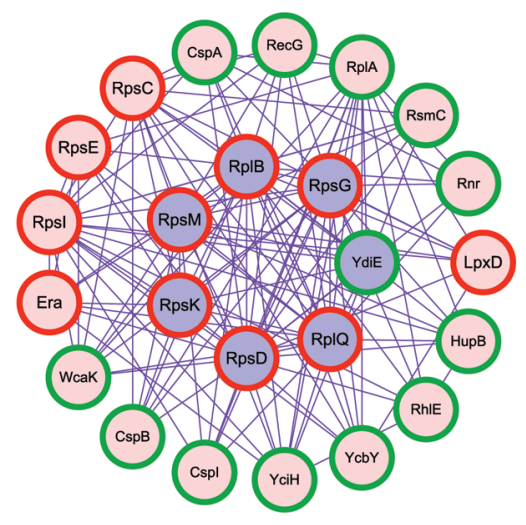

K6 nodes

K7 nodes

Essential

Nonessential

(B)

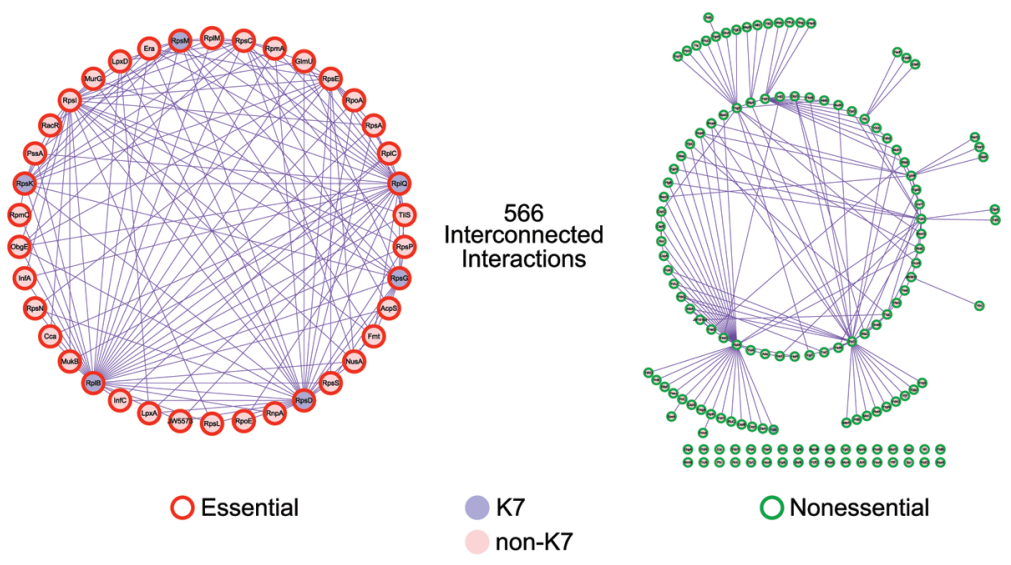

(C)

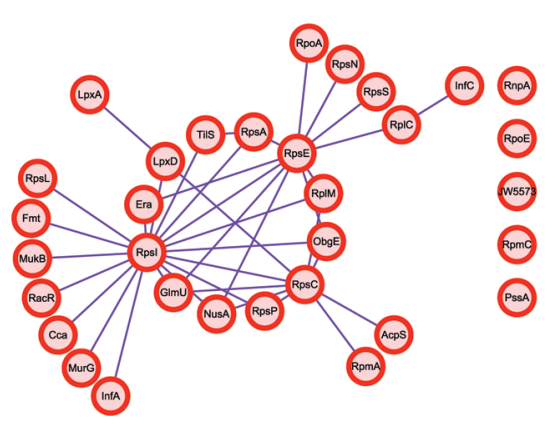

Figure 2. (A) A graphic representation of the subnetwork consisting of all $K_{6}$ cliques in E. coli. All of the $K_{6}$ cliques are associated with the maximum clique $K_{7}$. The seven nodes in $K_{7}$ are filled with blue color. Essential proteins are labeled with red circles and nonessential proteins with green circles. (B) Subnetworks of essential and nonessential proteins within the essential core. A graphic representation of the core subnetwork $C_{7}$ in $E$. coli after removal of the edges between essential (red nodes) and nonessential (green nodes) proteins. The essential proteins are tightly connected with each other (35 proteins and 137 interactions with density of 0.23 ), while the nonessential proteins are loosely connected with each other (127 proteins and 117 interactions with density of 0.01$)$. (C) Subnetwork of non- $K_{7}$ essential proteins within the essential core, that is, the subnetwork of $C_{7}^{\text {es }}$ after removal of the $K_{7}$ nodes. The subnetwork of essential proteins becomes quite sparse (32 proteins with 39 interactions and density of 0.08 ). The interactions between this subnetwork and the nonessential proteins in the essential core also decrease a lot from 566 to 177.

larger cliques is higher than smaller ones or nonclique proteins. We conclude that essential proteins tend to be part of larger cliques compared to nonessential proteins.

Characteristics of the Maximum Clique in $E$. coli. In this network of E. coli, there is a maximum clique with 7 nodes. In this clique, six nodes are essential and belong to ribosomal proteins, but the seventh node, YdiE, is a nonessential small protein with 63 amino acids. Since the function of YdiE is still unknown, we investigated YdiE further and listed the detailed information about its interacting partner proteins in Table 3. The degree of YdiE is 13, and there are 10 ribosomal proteins and 10 essential proteins among its interacting partners. The isoelectric point, $\mathrm{p} I$, of the 10 ribosomal proteins is approximately $9-11$, and the calculated $\mathrm{pI}$ of YdiE is 9.77 , similar to the $\mathrm{p} I$ values of ribosomal proteins. Therefore, we conjectured that YdiE may be a candidate of ribosomal proteins.

The centrality measurements may give us clues on the role of the $K_{7}$ clique. As shown in Table 1 , both the mean betweenness centrality and the mean closeness centrality of $K_{7}$-nodes are significantly higher than those of random samples of 7-node subnetworks (betweenness: 0.022 versus 0.00079 , with $Z$-score of 7.1 ; closeness: 0.37 versus $0.30, Z=4.2$ ). The higher the 


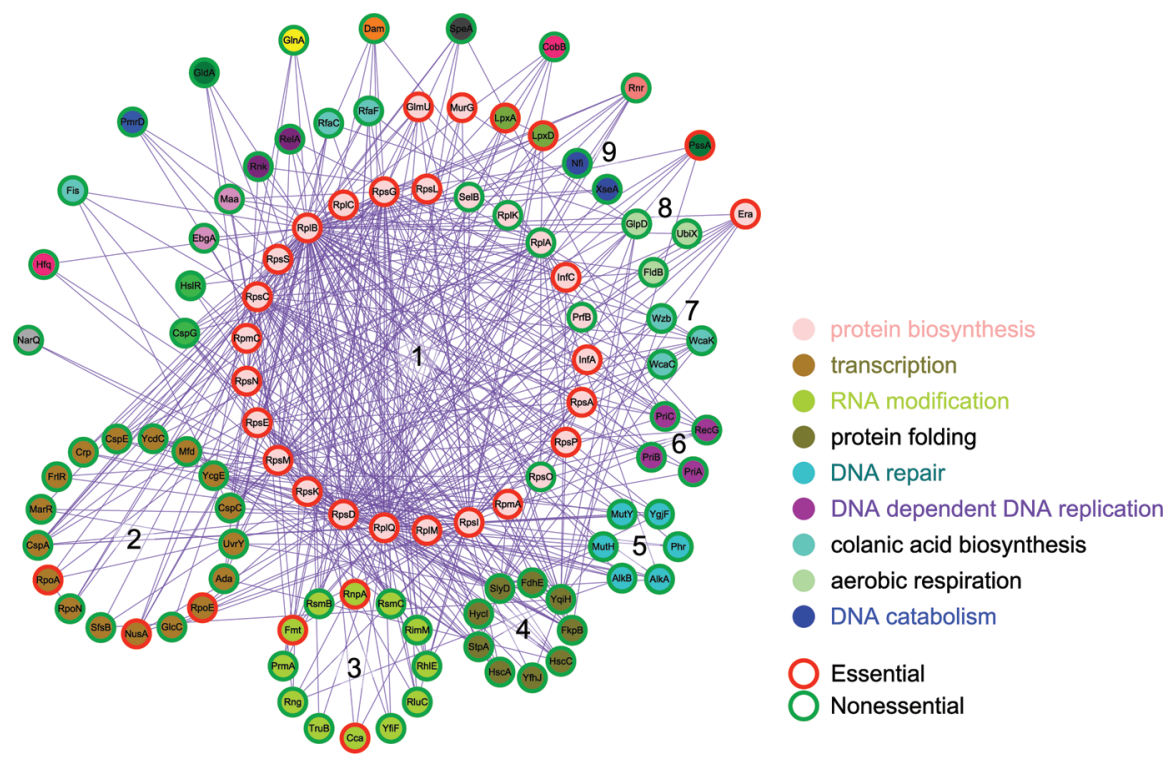

Figure 3. A graphic representation of the essential core $C_{7}$ in $E$. coli illustrating its biological functions. The proteins are clustered and labeled with different colors according to their annotated functional categories including (1) protein biosynthesis, (2) transcription, (3) RNA modification, (4) protein folding, (5) DNA repair, (6) colanic acid biosynthesis, (7) DNA-dependent DNA replication, (8) aerobic respiration, (9) DNA catabolism, and others. The border of an essential protein is colored as dark red, while that of a nonessential protein is colored as dark green.

betweenness centrality for a subnetwork, the more the shortest paths pass through the subnetwork, analogous to a traffic hub in a metropolitan area. Our results suggest that the $K_{7}$ clique plays a pivotal role in the protein interaction network. It is known that the central nodes in a network tend to have higher closeness centrality. Since $K_{7}$ has a significantly higher closeness centrality, it should be very close to the center in this network of $E$. coli. Furthermore, the mean path length between $K_{7}$ and the other nodes of the network is 2.12 (significantly shorter than the whole-network average of 3.35), and the eccentricity of $K_{7}$ is 5 , the same as the radius of the network. We may conclude that $K_{7}$ is the central module of the network.

There are $16 K_{6}$ cliques in the protein network of E. coli and they consist of 17 proteins, including all the nodes in $K_{7}$ (Figure $2 \mathrm{~A})$. This indicated that the 17 proteins themselves form a dense subnetwork associated with the largest clique $K_{7}$ in the network. To further expand this dense subnetwork, we proposed a clique-merge algorithm and used it to merge all the cliques closely associated with the $K_{7}$ clique.

Merging Maximum Clique with Neighboring Cliques Leads to a Dense and Essential Core. We merged $K_{7}$ with its overlapping neighboring cliques to form a larger cluster $C_{7}$ of 176 proteins and 864 interactions. If half or more nodes of a clique overlap with $K_{7}$, we defined this clique as a neighboring clique. Large-size cliques in the network, including all the $K_{6}$ and $K_{5}$ cliques, were merged into $C_{7}$. The density of $C_{7}$ is 0.06 , 30 times the average of randomly selected subnetworks with the same size ( 0.06 observed versus 0.002 expected, $Z=50, P$ $<0.0001)$. Furthermore, this dense cluster contains 38 essential proteins. Its essentiality is $22 \%$, which is significantly higher than the average of random subnetworks $(22 \%$ observed versus $8.5 \%$ expected, $Z=6.8, P<0.0001)$. Since there were no more clusters with similar size and featuring comparable density or essentiality, we called this cluster an essential core in the protein interaction network of E. coli.

When we removed the 566 interconnected interactions between essential and nonessential proteins in the cluster, the result showed that the subnetwork $C_{7}^{\text {es }}$, constructed solely by essential proteins, was very compact with density of 0.23 ; on the other hand, the one $C_{7}^{\text {ne }}$ constructed by nonessential proteins was quite sparse with density of 0.01 , as shown in Figure 2B. Furthermore, there were a total of 864 interactions in this dense cluster $C_{7}$ but 566 of them were interconnected interactions, implying that essential proteins and nonessential proteins are well connected. Among the rest of the 298 interactions, 146 of them were shared by 38 essential nodes, the other 152 interactions were shared by 138 nonessential nodes. This means that essential proteins tend to form a tighter cluster while nonessential proteins play auxiliary roles to essential proteins. When we further removed $K_{7}$ nodes out of the subnetwork $C_{7}^{\text {es }}$, the subnetwork collapsed and became sparser (32 proteins with 39 interactions and density of 0.08 ), as shown in Figure 2C. We conclude that $K_{7}$ nodes play important roles in the subnetwork constructed by essential proteins and take part in most of the interconnected interactions.

To illustrate the biological functions involving the essential core $C_{7}$, we categorized the proteins by their functional annotations in Gene Ontology ${ }^{32}$ and visualized their interaction subnetworks accordingly, as shown in Figure 3. The major function of this essential core is protein biosynthesis as it contains many ribosomal proteins. Other proteins in the core are involved in closely related biological functions such as transcription, RNA modification, and protein folding.

Clique Analysis of $\boldsymbol{S}$. cerevisiae Network. To examine whether the observed characteristics of cliques in E. coli exist in other organisms, we applied the clique analysis to $S$. cerevisiae, the well-studied budding yeast, and found that its protein interaction network shows similar behavior to $E$. coli. As shown in Figure 1B, a strong correlation is observed between clique size and essentiality in yeast. The maximum clique in yeast has a size of 10 and contains extremely large proportion of essential proteins, $90 \%$.

In the same way, we calculated the centrality of the maximum clique, $K_{10}$, in the protein interaction network of yeast. 
Both the betweenness and closeness centrality of $K_{10}$ nodes were significantly higher than other nodes in the network (average betweenness: 0.0022 versus $0.0006, P<6.55 \times 10^{-6}$; average closeness: 0.29 versus $0.24, P<6.49 \times 10^{-7}$ ). On the other hand, the average path length between the $K_{10}$ nodes and essential proteins was significantly shorter than the path length between $K_{10}$ and nonessential proteins (2.65 versus $2.87, P<$ $1.11 \times 10^{-13}$ ), indicating that $K_{10}$ nodes are significantly closer to essential proteins than nonessential ones.

Merging the maximum clique $K_{10}$ with overlapping neighboring cliques also gives rise to a dense cluster $C_{10}$ with high essentiality, similar to the essential core in E. coli. $C_{10}$ consists of 42 proteins with 23 essential and 241 interactions, mainly involved in protein catabolic process. However, the size of $C_{10}$ was not large, as not all larger cliques merged with $C_{10}$; instead, they merged with other cliques to form various dense and essential clusters.

Comparing the network of E. coli and S. cerevisiae by the proposed clique analysis, we found that there is one essential core in E. coli, but probably more than one essential core in $S$. cerevisiae (shown in Supporting Information S3). This difference might be due to the fact that $E$. coli is a prokaryote, and $S$. cerevisiae is a eukaryote which has more complex cellular structure. On the other hand, the observed difference might be the consequence of incomplete protein-protein interaction measurements. We performed the analysis on another largescale protein-protein interaction data set of $E$. $\operatorname{coli}^{6}$ and observed similar results (Supporting Information S4). With the increasing effort in the systematic measurements of various model organisms, we will be able to investigate whether similar essential core structure exists in other organisms and how the protein-protein interaction network is organized from the aspect of essential cores.

Wuchty and Almaas ${ }^{26}$ found that, by applying $k$-core decomposition on protein interaction network, the probability of nodes being both essential and evolutionary conserved successively increases toward the innermost cores. A $k$-core means a subnetwork of all the nodes with degree higher than $k$. The large cliques or the merged clique clusters in our study are topologically correlated with high $k$-cores. Therefore, our observations of the relationship between clique size and essentiality, as well as our proposed essential cores are consistent with the findings of the $k$-core analysis. High $k$-cores (innermost cores) might represent an alternative form of essential cores. On the other hand, our clique analysis method could be viewed as a new approach to network core decomposition. Recently, Zotenko et al. $^{33}$ analyzed the protein interaction network of $S$. cerevisiae and demonstrated that a majority of hubs are essential due to their involvement in a group of densely connected proteins with shared biological function that are enriched in essential proteins. In this work, we analyzed the protein interaction networks of $E$. coli as well as $S$. cerevisiae. We used a novel approach to identify a group of densely connected proteins, which were found to share similar biological functions and have high essentiality. Our study provides complementary analysis and results not addressed in previous studies.

\section{Conclusions}

On the basis of our analysis, we found that essential proteins tend to be a part of larger cliques than nonessential proteins in $E$. coli and yeast. Larger cliques consist of a greater proportion of essential proteins. In E. coli, there exists a maximum clique of size 7 , and the ratio of essential proteins to all proteins in this maximum clique is $86 \%$, or $6 / 7$. Similarly, there exists a maximum clique of size 10 in yeast, and the ratio is $90 \%$, or $9 / 10$. We showed that a maximum clique with extremely high ratios of essential proteins is present in the protein interaction networks of $E$. coli and yeast. Considering other topological properties, such as closeness centrality, betweenness centrality, and path length, this maximum clique was shown to play a central role in the network. Expanding the maximum clique by merging neighboring cliques, we discovered a dense cluster that contains a significantly higher ratio of essential proteins, and we defined it as an essential core of the protein interaction network. These properties are shared by both $E$. coli and yeast; hence, there might be some common rules of thumb between organisms in their protein interaction network structure. The existence of essential cores may provide a different aspect to organizing the protein interaction network into models of cell machinery.

Acknowledgment. This work was supported by the National Science Council of Taiwan, the National Science and Technology Program for Agricultural Biotechnology from Council of Agriculture in Taiwan, CREST, JST and a Grant-in-Aid for Scientific Research on Priority Areas from the Ministry of Education, Culture, Sports, Science and Technology of Japan. We thank Jason Lee for proofreading the manuscript.

Supporting Information Available: Dependence of clustering coefficients on possible false positive interactions in E. coli; network analysis methods; supplementary results of clique-merging; additional protein-protein interaction data for E. coli. This material is available free of charge via the Internet at http://pubs.acs.org.

\section{References}

(1) Fields, S.; Song, O. A novel genetic system to detect protein-protein interactions. Nature 1989, 340 (6230), 245-6.

(2) Mann, M.; Hendrickson, R. C.; Pandey, A. Analysis of proteins and proteomes by mass spectrometry. Annu. Rev. Biochem. 2001, 70, 437-73.

(3) Ito, T.; Chiba, T.; Ozawa, R.; Yoshida, M.; Hattori, M.; Sakaki, Y. A comprehensive two-hybrid analysis to explore the yeast protein interactome. Proc. Natl. Acad. Sci. U.S.A. 2001, 98 (8), 4569-74.

(4) Uetz, P.; Giot, L.; Cagney, G.; Mansfield, T. A.; Judson, R. S.; Knight, J. R.; Lockshon, D.; Narayan, V.; Srinivasan, M.; Pochart, P.; Qureshi-Emili, A.; Li, Y.; Godwin, B.; Conover, D.; Kalbfleisch, T.; Vijayadamodar, G.; Yang, M.; Johnston, M.; Fields, S.; Rothberg, J. M. A comprehensive analysis of protein-protein interactions in Saccharomyces cerevisiae. Nature 2000, 403 (6770), 623-7.

(5) Arifuzzaman, M.; Maeda, M.; Itoh, A.; Nishikata, K.; Takita, C.; Saito, R.; Ara, T.; Nakahigashi, K.; Huang, H. C.; Hirai, A.; Tsuzuki, K.; Nakamura, S.; Altaf-Ul-Amin, M.; Oshima, T.; Baba, T.; Yamamoto, N.; Kawamura, T.; Ioka-Nakamichi, T.; Kitagawa, M.; Tomita, M.; Kanaya, S.; Wada, C.; Mori, H. Large-scale identification of protein-protein interaction of Escherichia coli K-12. Genome Res. 2006, 16 (5), 686-91.

(6) Butland, G.; Peregrin-Alvarez, J. M.; Li, J.; Yang, W.; Yang, X.; Canadien, V.; Starostine, A.; Richards, D.; Beattie, B.; Krogan, N.; Davey, M.; Parkinson, J.; Greenblatt, J.; Emili, A. Interaction network containing conserved and essential protein complexes in Escherichia coli. Nature 2005, 433 (7025), 531-7.

(7) Tornow, S.; Mewes, H. W. Functional modules by relating protein interaction networks and gene expression. Nucleic Acids Res. 2003, 31 (21), 6283-9.

(8) Han, J. D.; Bertin, N.; Hao, T.; Goldberg, D. S.; Berriz, G. F.; Zhang, L. V.; Dupuy, D.; Walhout, A. J.; Cusick, M. E.; Roth, F. P.; Vidal, M. Evidence for dynamically organized modularity in the yeast protein-protein interaction network. Nature 2004, 430 (6995), 8893.

(9) Winzeler, E. A.; Shoemaker, D. D.; Astromoff, A.; Liang, H.; Anderson, K.; Andre, B.; Bangham, R.; Benito, R.; Boeke, J. D.; 
Bussey, H.; Chu, A. M.; Connelly, C.; Davis, K.; Dietrich, F.; Dow, S. W.; El Bakkoury, M.; Foury, F.; Friend, S. H.; Gentalen, E.; Giaever, G.; Hegemann, J. H.; Jones, T.; Laub, M.; Liao, H.; Liebundguth, N.; Lockhart, D. J.; Lucau-Danila, A.; Lussier, M.; M’Rabet, N.; Menard, P.; Mittmann, M.; Pai, C.; Rebischung, C.; Revuelta, J. L.; Riles, L.; Roberts, C. J.; Ross-MacDonald, P.; Scherens, B.; Snyder, M.; Sookhai-Mahadeo, S.; Storms, R. K.; Veronneau, S.; Voet, M.; Volckaert, G.; Ward, T. R.; Wysocki, R.; Yen, G. S.; Yu, K.; Zimmermann, K.; Philippsen, P.; Johnston, M.; Davis, R. W. Functional characterization of the S. cerevisiae genome by gene deletion and parallel analysis. Science 1999, 285 (5429), 901-6.

(10) Baba, T.; Ara, T.; Hasegawa, M.; Takai, Y.; Okumura, Y.; Baba, M.; Datsenko, K. A.; Tomita, M.; Wanner, B. L.; Mori, H. Construction of Escherichia coli K-12 in-frame, single-gene knockout mutants: the Keio collection. Mol. Syst. Biol. 2006, 2, 2006-0008.

(11) Kobayashi, K.; Ehrlich, S. D.; Albertini, A.; Amati, G.; Andersen, K. K.; Arnaud, M.; Asai, K.; Ashikaga, S.; Aymerich, S.; Bessieres, P.; Boland, F.; Brignell, S. C.; Bron, S.; Bunai, K.; Chapuis, J.; Christiansen, L. C.; Danchin, A.; Debarbouille, M.; Dervyn, E.; Deuerling, E.; Devine, K.; Devine, S. K.; Dreesen, O.; Errington, J.; Fillinger, S.; Foster, S. J.; Fujita, Y.; Galizzi, A.; Gardan, R.; Eschevins, C.; Fukushima, T.; Haga, K.; Harwood, C. R.; Hecker, M.; Hosoya, D.; Hullo, M. F.; Kakeshita, H.; Karamata, D.; Kasahara, Y.; Kawamura, F.; Koga, K.; Koski, P.; Kuwana, R.; Imamura, D.; Ishimaru, M.; Ishikawa, S.; Ishio, I.; Le Coq, D.; Masson, A.; Mauel, C.; Meima, R.; Mellado, R. P.; Moir, A.; Moriya, S.; Nagakawa, E.; Nanamiya, H.; Nakai, S.; Nygaard, P.; Ogura, M.; Ohanan, T.; O’Reilly, M.; O’Rourke, M.; Pragai, Z.; Pooley, H. M.; Rapoport, G.; Rawlins, J. P.; Rivas, L. A.; Rivolta, C.; Sadaie, A.; Sadaie, Y.; Sarvas, M.; Sato, T.; Saxild, H. H.; Scanlan, E.; Schumann, W.; Seegers, J. F.; Sekiguchi, J.; Sekowska, A.; Seror, S. J.; Simon, M.; Stragier, P.; Studer, R.; Takamatsu, H.; Tanaka, T.; Takeuchi, M.; Thomaides, H. B.; Vagner, V.; van Dijl, J. M.; Watabe, K.; Wipat, A.; Yamamoto, H.; Yamamoto, M.; Yamamoto, Y.; Yamane, K.; Yata, K.; Yoshida, K.; Yoshikawa, H.; Zuber, U.; Ogasawara, N. Essential Bacillus subtilis genes. Proc. Natl. Acad. Sci. U.S.A. 2003, 100 (8), 4678-83.

(12) Giaever, G.; Chu, A. M.; Ni, L.; Connelly, C.; Riles, L.; Veronneau, S.; Dow, S.; Lucau-Danila, A.; Anderson, K.; Andre, B.; Arkin, A. P.; Astromoff, A.; El-Bakkoury, M.; Bangham, R.; Benito, R.; Brachat, S.; Campanaro, S.; Curtiss, M.; Davis, K.; Deutschbauer, A.; Entian, K. D.; Flaherty, P.; Foury, F.; Garfinkel, D. J.; Gerstein, M.; Gotte, D.; Guldener, U.; Hegemann, J. H.; Hempel, S.; Herman, Z.; Jaramillo, D. F.; Kelly, D. E.; Kelly, S. L.; Kotter, P.; LaBonte, D.; Lamb, D. C.; Lan, N.; Liang, H.; Liao, H.; Liu, L.; Luo, C.; Lussier, M.; Mao, R.; Menard, P.; Ooi, S. L.; Revuelta, J. L.; Roberts, C. J.; Rose, M.; Ross-Macdonald, P.; Scherens, B.; Schimmack, G.; Shafer, B.; Shoemaker, D. D.; Sookhai-Mahadeo, S.; Storms, R. K.; Strathern, J. N.; Valle, G.; Voet, M.; Volckaert, G.; Wang, C. Y.; Ward, T. R.; Wilhelmy, J.; Winzeler, E. A.; Yang, Y.; Yen, G.; Youngman, E.; Yu, K.; Bussey, H.; Boeke, J. D.; Snyder, M.; Philippsen, P.; Davis, R. W.; Johnston, M. Functional profiling of the Saccharomyces cerevisiae genome. Nature 2002, 418 (6896), 387-91.

(13) Jeong, H.; Mason, S. P.; Barabasi, A. L.; Oltvai, Z. N. Lethality and centrality in protein networks. Nature 2001, 411 (6833), 41-2.

(14) Yook, S. H.; Oltvai, Z. N.; Barabasi, A. L. Functional and topological characterization of protein interaction networks. Proteomics 2004, 4 (4), 928-42.

(15) Reguly, T.; Breitkreutz, A.; Boucher, L.; Breitkreutz, B. J.; Hon, G. C.; Myers, C. L.; Parsons, A.; Friesen, H.; Oughtred, R.; Tong, A.; Stark, C.; Ho, Y.; Botstein, D.; Andrews, B.; Boone, C.; Troyanskya, O. G.; Ideker, T.; Dolinski, K.; Batada, N. N.; Tyers, M. Comprehensive curation and analysis of global interaction networks in Saccharomyces cerevisiae. J. Biol. 2006, 5 (4), 11.

(16) Joy, M. P.; Brock, A.; Ingber, D. E.; Huang, S. High-betweenness proteins in the yeast protein interaction network. J. Biomed. Biotechnol. 2005, 2005 (2), 96-103.

(17) Krogan, N. J.; Cagney, G.; Yu, H.; Zhong, G.; Guo, X.; Ignatchenko, A.; Li, J.; Pu, S.; Datta, N.; Tikuisis, A. P.; Punna, T.; Peregrin-
Alvarez, J. M.; Shales, M.; Zhang, X.; Davey, M.; Robinson, M. D.; Paccanaro, A.; Bray, J. E.; Sheung, A.; Beattie, B.; Richards, D. P.; Canadien, V.; Lalev, A.; Mena, F.; Wong, P.; Starostine, A.; Canete, M. M.; Vlasblom, J.; Wu, S.; Orsi, C.; Collins, S. R.; Chandran, S.; Haw, R.; Rilstone, J. J.; Gandi, K.; Thompson, N. J.; Musso, G.; St Onge, P.; Ghanny, S.; Lam, M. H.; Butland, G.; Altaf-Ul, A. M.; Kanaya, S.; Shilatifard, A.; O'Shea, E.; Weissman, J. S.; Ingles, C. J.; Hughes, T. R.; Parkinson, J.; Gerstein, M.; Wodak, S. J.; Emili, A.; Greenblatt, J. F. Global landscape of protein complexes in the yeast Saccharomyces cerevisiae. Nature 2006, 440 (7084), 637-43.

(18) Coulomb, S.; Bauer, M.; Bernard, D.; Marsolier-Kergoat, M. C. Gene essentiality and the topology of protein interaction networks. Proc. Biol. Sci. 2005, 272 (1573), 1721-5.

(19) He, X.; Zhang, J. Why do hubs tend to be essential in protein networks. PLoS Genet. 2006, 2 (6), e88.

(20) Gandhi, T. K.; Zhong, J.; Mathivanan, S.; Karthick, L.; Chandrika, K. N.; Mohan, S. S.; Sharma, S.; Pinkert, S.; Nagaraju, S.; Periaswamy, B.; Mishra, G.; Nandakumar, K.; Shen, B.; Deshpande, N.; Nayak, R.; Sarker, M.; Boeke, J. D.; Parmigiani, G.; Schultz, J.; Bader, J. S.; Pandey, A. Analysis of the human protein interactome and comparison with yeast, worm and fly interaction datasets. Nat. Genet. 2006, 38 (3), 285-93.

(21) Barabasi, A. L.; Oltvai, Z. N. Network biology: understanding the cell's functional organization. Nat. Rev. Genet. 2004, 5 (2), 10113.

(22) Hartwell, L. H.; Hopfield, J. J.; Leibler, S.; Murray, A. W. From molecular to modular cell biology. Nature 1999, 402 (6761 Suppl), C4752.

(23) Rives, A. W.; Galitski, T. Modular organization of cellular networks. Proc. Natl. Acad. Sci. U.S.A. 2003, 100 (3), 1128-33.

(24) Parrish, J. R.; Yu, J.; Liu, G.; Hines, J. A.; Chan, J. E.; Mangiola, B. A.; Zhang, H.; Pacifico, S.; Fotouhi, F.; DiRita, V. J.; Ideker, T.; Andrews, P.; Finley, R. L. A proteome-wide protein interaction map for Campylobacter jejuni. Genome Biol. 2007, 8 (7), R130.

(25) Tong, A. H.; Drees, B.; Nardelli, G.; Bader, G. D.; Brannetti, B.; Castagnoli, L.; Evangelista, M.; Ferracuti, S.; Nelson, B.; Paoluzi, S.; Quondam, M.; Zucconi, A.; Hogue, C. W.; Fields, S.; Boone, C.; Cesareni, G. A combined experimental and computational strategy to define protein interaction networks for peptide recognition modules. Science 2002, 295 (5553), 321-4.

(26) Wuchty, S.; Almaas, E. Peeling the yeast protein network. Proteomics 2005, 5 (2), 444-9.

(27) Deane, C. M.; Salwinski, L.; Xenarios, I.; Eisenberg, D. Protein interactions: two methods for assessment of the reliability of high throughput observations. Mol. Cell. Proteomics 2002, 1 (5), 34956.

(28) Chua, H. N.; Sung, W. K.; Wong, L. Exploiting indirect neighbours and topological weight to predict protein function from proteinprotein interactions. Bioinformatics 2006, 22 (13), 1623-30.

(29) Pemmaraju, S.; Skiena, S. Computational Discrete Mathematics: Combinatorics and Graph Theory with Mathematica; Cambridge University Press: Cambridge, England, 2003.

(30) Yu, H.; Greenbaum, D.; Xin Lu, H.; Zhu, X.; Gerstein, M. Genomic analysis of essentiality within protein networks. Trends Genet. 2004, 20 (6), 227-31.

(31) Kim, P. M.; Lu, L. J.; Xia, Y.; Gerstein, M. B. Relating threedimensional structures to protein networks provides evolutionary insights. Science 2006, 314 (5807), 1938-41.

(32) Ashburner, M.; Ball, C. A.; Blake, J. A.; Botstein, D.; Butler, H.; Cherry, J. M.; Davis, A. P.; Dolinski, K.; Dwight, S. S.; Eppig, J. T.; Harris, M. A.; Hill, D. P.; Issel-Tarver, L.; Kasarskis, A.; Lewis, S.; Matese, J. C.; Richardson, J. E.; Ringwald, M.; Rubin, G. M.; Sherlock, G. Gene ontology: tool for the unification of biology. The Gene Ontology Consortium. Nat. Genet. 2000, 25 (1), 25-9.

(33) Zotenko, E.; Mestre, J.; O'Leary, D. P.; Przytycka, T. M. Why do hubs in the yeast protein interaction network tend to be essential: reexamining the connection between the network topology and essentiality. PLoS Comput. Biol. 2008, 4 (8), e1000140.
PR8008786 